\title{
COMPORTAMENTO IMPULSIVO: ESTUDO EM UMA POPULAÇÃO DE UNIVERSITÁRIOS
}

\author{
IMPULSIVE BEHAVIOR: A STUDY IN A GROUP OF UNIVERSITY STUDENTS
}

\author{
Silvia Rosane Parcias ${ }^{\mathrm{a}^{*}}$, Lyara Schaefer Sombrio ${ }^{\mathrm{b}^{*}}$, Nayara Teixeira Flügel ${ }^{\mathrm{c}^{*}}$, Maria Julia Parcias \\ do Rosario ${ }^{\mathrm{d}^{*}}$, Melissa de Carvalho Souza ${ }^{\mathrm{e}^{* *}}$, Adriana Coutinho de Azevedo Guimarães ${ }^{\text {* }^{* *}}$ \\ asil_pa13@hotmail.com, bly_ss@hotmail.com, cnaflugel@hotmail.com, djujurosario@hotmail.com, \\ emecarvalho.s@gmail.com, fnanaguim@terra.com.br \\ *Universidade do Sul de Santa Catarina - Palhoça (SC), Brasil \\ ${ }^{* *}$ Universidade do Estado de Santa Catarina - Florianópolis (SC), Brasil
}

Data de entrada do artigo: 26/05/2013

Data de aceite do artigo: 08/05/2014

\section{RESUMO}

O estudo de corte transversal objetivou investigar o comportamento impulsivo de estudantes universitários de uma universidade do sul do Brasil. A amostra foi constituída por 129 estudantes dos cursos de Direito e Engenharia Civil, sendo $49,6 \%$ do sexo masculino e 50,4\% do sexo feminino. Optou-se por um questionário autoaplicável composto por três partes: a) identificação pessoal; b) situação socioeconômica; e c) escala de impulsividade de Barratt 11. A análise dos dados foi feita por meio da estatística descritiva (frequência simples, percentual, média e desvio padrão) e inferencial pelo teste do qui-quadrado para comparação entre os resultados. A maioria dos estudantes está nas fases iniciais dos cursos (69,8\%), sendo que $57,4 \%$ cursam Engenharia Civil enquanto 42,6\% Direito, e pertencem aos estratos econômicos A e B (88,3\%). Quanto ao índice de impulsividade, verificou-se que a maior parte dos participantes está dentro dos limites normais (69\%). Na comparação entre o comportamento impulsivo e as variáveis sociodemográficas não houve diferença estatística significativa.

Palavras-chave: Comportamento impulsivo; estudantes; saúde.

\section{ABSTRACT}

The cross-sectional study aimed to investigate the impulsive behavior among university students of a university in Southern Brazil. The sample was composed of 129 students of Law and Civil Engineering courses, $49.6 \%$ male and $50.4 \%$ female. A self-applicable questionnaire, which was composed of three parts: a) personal identification; b) socioeconomic situation and c) Barratt Impulsiveness Scale - BIS 11 was chosen. The data were analyzed through descriptive (simple frequency, percentage, mean and standard deviation) and inferential statistic using the qui-square to compare the results. Most of the students are in the beginning of their undergraduate courses (69.8\%), 57.4\% study Civil Engineering, and $42.6 \%$ study Law, belonging to the economic status A and B (88.3\%). As for the impulsive level, most of the participants are within normal limits (69\%). No significance was found in the comparison of impulsive behavior and demographic variables.

Keywords: Impulsive behavior; students; health 


\section{Introdução}

A impulsividade pode ser percebida como uma decisão adotada sem avaliar todas as suas implicaçóes e as possíveis consequências do comportamento impulsivo. É um tema a ser analisado que merece evidência, haja visto que atinge pelo menos metade da humanidade em algum período da vida, no qual eventos, por menores que sejam, podem acarretar em uma máxima reação do ponto de vista emocional ${ }^{1}$. É considerado um fenótipo complexo, definido por diferentes padróes cognitivos e comportamentais, podendo levar a consequências disfuncionais tanto imediatas quanto médio/longo prazos².

O comportamento impulsivo acontece no momento em que existem mudanças no decurso da açáo sem que seja feita uma avaliaçáo prévia e consciente, ou quando ocorrem comportamentos precipitados, ou ainda, quando se manifesta uma inclinação a agir com um nível de planejamento menor em comparação a indivíduos de nível intelectual semelhante ${ }^{2}$. Os indivíduos impulsivos tendem a mostrar falta de previsão das consequências de seus atos ${ }^{1,3}$. A falta de controle emocional leva o indivíduo a tomar atitudes sem um exame atento das implicaçóes e os possíveis resultados do comportamento em busca de satisfação imediata do desejo ${ }^{4}$. Muitos são os problemas que este tipo de comportamento pode trazer às pessoas, como dificuldades no emprego e na convivência familiar, problemas financeiros por gastos em excesso e, até mesmo, comprometimento de sua própria saúde .

O mesmo comportamento inclui características biológicas e hereditárias ${ }^{6}$, podendo ocasionar impactos e prejuízos em diversas situaçóes do cotidiano. Tem sido frequentemente associado tanto a comportamentos de risco em geral quanto a violaçóes da lei de trânsito e acidentes automobilísticos ${ }^{3,7}$. Há também uma associação entre impulsividade e aumento na chance de consumo abusivo de substâncias psicoativas ${ }^{8}$. Constata-se que os indivíduos mais impulsivos têm cerca de três vezes mais probabilidades de tornarem-se consumidores problemáticos dessas substâncias?.

Há diversos neurotransmissores que podem estar envolvidos no acontecimento do comportamento impulsivo ${ }^{10}$, sendo que cada vez mais novos transtornos são atribuídos a problemas com esse tipo de comportamento, como jogo patológico e transtornos alimentares ${ }^{11}$.

Normalmente a maioria dos seres humanos possui certo grau de impulsividade, que pode ser considerada um traço de personalidade, por englobar características pessoais como espontaneidade, criatividade, rapidez de resposta, precipitação e desorganização ${ }^{7}$. Entretanto, diversos são os transtornos em que as diferentes manifestaçôes da impulsividade se apresentam de forma intensa, gerando prejuízos para o indivíduo e aqueles que o cercam. Tais transtornos são do controle do impulso e de personalidade, além de outros, descritos nos eixos I e II do Manual Diagnóstico e Estatístico de Transtornos Mentais - DSM-IV ${ }^{12}$.

Alguns estudos com universitários encontraram pontuação mais alta para impulsividade nos indivíduos com relato de mais envolvimento em acidentes ${ }^{3,13} \mathrm{e}$ também mostraram que o comportamento impulsivo sinergicamente aumenta a chance de problemas com álcool ${ }^{14}$. Apesar de estudos ${ }^{9,10,14}$ mostrarem que a impulsividade pode estar associada a transtornos relacionados ao álcool e outras drogas, há uma carência de evidências em amostras comunitárias, particularmente em adolescentes ${ }^{15}$.

Os estudos citados justificam a escolha desta população, ou seja, de universitários, no qual se observa características individuais quanto à impulsividade tanto em homens quanto em mulheres, podendo ser considerados influentes fatores para os comportamentos de risco à saúde em indivíduos adultos joven ${ }^{16}$.

Nomeadamente, a escolha do tema deu-se da importância e da necessidade de avaliar os dados sociodemográficos e impulsividade, com intuito de fomentar o conhecimento, contribuindo para um melhor entendimento de alguns aspectos da impulsividade, como as manifestaçóes clínicas do comportamento impulsivo. Além do quê, o estudo utilizou como base a Escala de Impulsividade de Barrat, que é muito pertinente para a prática médica, por sua comprovada utilidade para a análise psiquiátrica em muitos países ao redor do mundo e por se tratar de uma escala recentemente validada para o cotidiano brasileiro ${ }^{2}$. Sendo assim, objetivou-se investigar o comportamento impulsivo de estudantes de uma universidade do sul do Brasil.

\section{Métodos}

O estudo de corte transversal foi aprovado pelo Comitê de Ética em Pesquisa da Universidade do Sul de Santa Catarina (CEP-UNISUL), protocolo no 11.355.4.01.III. Todos os participantes assinaram o Termo de Consentimento Livre e Esclarecido.

Com uma populaçáo de cerca de 210 estudantes, matriculados nos cursos de graduação de Direito e Engenharia Civil da Universidade do Sul de Santa Catarina (UNISUL) - Campus Pedra Branca, localizado na Grande Florianópolis, no segundo semestre de 2011, nos turnos matutino, vespertino e noturno. A amostra não probabilística intencional foi composta por 129 estudantes de ambos os gêneros, matriculados nas duas primeiras e nas duas últimas fases dos cursos.

A UNISUL é uma universidade privada, situada no estado de Santa Catarina, sendo uma instituição educacional multicampi. 
A escolha destes dois cursos foi determinada pela acessibilidade em função da distribuição da grade horária e da permanência dos alunos investigados no campus durante a fase de realização desta pesquisa.

Os fatores de inclusão foram: estar cursando somente um curso de graduação universitário e estar matriculado nas duas primeiras ou nas duas últimas fases de seu curso.

A coleta de dados aconteceu nos centros respectivos de cada curso, durante o horário de aula, sendo o questionário autoaplicável respondido individualmente, em torno de 15 minutos, com a orientação do pesquisador, esclarecendo dúvidas sobre o seu correto preenchimento.

O questionário autoaplicável foi composto de três partes:

a) identificação pessoal - questôes destinadas a identificar sexo, idade, escolaridade e fase do aluno;

b) situação socioeconômica - por meio do instrumento adotado pela Associação Nacional de Empresa de Pesquisa - Critério de Classificação Econômica Brasil (ABEP, 2010), que classifica a população em classes econômicas A, B, C, D e E, pela pontuação obtida e renda mensal ${ }^{17}$. Para fins estatísticos, agruparam-se os alunos do estrato D e E; e

c) Escala de Impulsividade de Barratt 11 (Barratt Impulsiveness Scale - BIS 11) - um dos métodos mais utilizados e influentes para avaliação da impulsividade segundo a literatura ${ }^{2,15,18}$, validada e adaptada para aplicação em adultos brasileiros em $2010^{2}$. Em estudos, foram obtidas evidências de validade ${ }^{2,15,18}$, sendo uma escala de autopreenchimento composta por 30 itens que foram classificados de acordo com uma escala do tipo Likert de quatro pontos, a saber: 1 = raramente ou nunca; 2 = de vez em quando; 3 = com frequência; $4=$ quase sempre/sempre. A pontuação varia de 30 a 120 pontos, e altos escores indicam a presença de comportamentos impulsivos. A escala classifica indivíduos com pontuaçáo $<52$ como muito controlados, de 52-71 pontos com limites normais de impulsividade, $\geq 72$ como altamente impulsivos ${ }^{2}$.

Além de um escore global, a BIS 11 permite o cálculo de escores parciais referentes a três subdomínios da impulsividade, sendo eles a impulsividade motora (itens $2,3,4,16,17,19,21,22,23,25$ e $30^{*}$ ), atencional (itens $6,5,9^{*}, 11,20^{*}, 24,26,28$ ) e por não planejamento (itens $1^{*}, 7^{*}, 8^{*}, 10^{*}, 12^{*}, 13^{*}, 14,15^{*}$, $\left.18,27,29^{*}\right)$; sendo que os itens marcados com o sinal $\left.{ }^{*}\right)$ recebem escore inverso para o cálculo dos escores parciais e total $(4,3,2,1)$. Os resultados das análises quantitativas indicam que a versão final do instrumento é satisfatória, sendo a BIS 11 uma escala útil para a análise psiquiátrica².

A análise dos dados foi realizada no programa estatístico SPSS - versão 17.0, por meio da estatística descritiva (frequência simples, percentual, média e desvio padrão) e inferencial, optando-se pelo teste do qui-quadrado para comparaçáo entre os resultados da BIS 11, isto é, entre o comportamento impulsivo e as variáveis sociodemográficas, com nível de significância de $95 \%$.

\section{Resultados}

A amostra foi constituída por 129 estudantes dos cursos de Direito e Engenharia Civil da UNISUL, representados por 55 e 74 alunos respectivamente. Participaram do estudo, 64 estudantes do sexo masculino, correspondendo a $49,6 \%$ da amostra, e 65 do sexo feminino, correspondendo a 50,4\%, com média de idade de 23,67 anos.

Em relação às fases, 69,8\% estavam em fases iniciais ( $1^{\underline{a}}$ e $2^{\underline{a}}$ fase) e $30,2 \%$ em fases finais (penúltima e última fase).

Segundo a estratificação da amostra por classes sociais, a maioria dos estudantes correspondeu à classe $\mathrm{B}$, com $61,2 \%$, enquanto as classes $\mathrm{A}, \mathrm{C}$ e $\mathrm{D}$ corresponderam a 27,$1 ; 10,9$ e $8 \%$ respectivamente. Na tabela 1 , encontram-se as características sociodemográficas dos participantes do estudo.

Tabela 1: Características dos participantes do estudo de acordo com as variáveis sociodemográficas

\begin{tabular}{ccc}
\hline Variáveis & n & $\%$ \\
\hline Sexo & & \\
\hline Masculino & 64 & 49,6 \\
Feminino & 65 & 50,4 \\
\hline Graduaçáo & & \\
\hline Direito & 55 & 42,6 \\
Engenharia Civil & 74 & 57,4 \\
\hline Fase & & \\
\hline Fases iniciais & 90 & 69,8 \\
Fases finais & 39 & 30,2 \\
\hline Estrato & & \\
Econômico & & \\
\hline Classe A & 35 & 27,1 \\
Classe B & 79 & 61,2 \\
Classe C & 15 & 11,7 \\
Total & 129 & 100 \\
\hline
\end{tabular}

Em relação à Escala de Impulsividade de Barrat versão $11,69 \%$ dos estudantes obtiveram pontuação entre 52 e 71 pontos, enquanto $31 \%$ pontuaram 72 ou mais.

A média de pontuação total da BIS 11 foi de 69,05 pontos, 23,87 correspondendo ao escore motor, 22,43 ao escore atencional e 30,5 pontos ao escore falta de planejamento. 
As médias referentes ao sexo masculino foram 23,53 para idade, 69,02 pontos para escore total da BIS 11, sendo que as médias de escore motor, atencional e falta de planejamento foram respectivamente 24; 22,02; e 30,34 pontos. No sexo feminino, encontramos uma idade média de 23,8 , enquanto as médias de escore motor, atencional e falta de planejamento foram respectivamente de 23,$74 ; 22,83$; e 30,65 pontos respectivamente, sendo o escore total da BIS 11 de 69,09 pontos.

$\mathrm{O}$ curso de Direito apresentou pontuação total da BIS 11 com média de 69,18 pontos, com escores motor, atencional e falta de planejamento com média de 23,98; 22,33; e 30,25 pontos respectivamente. Enquanto o curso de Engenharia Civil apresentou pontuaçáo total da BIS 11 com média de 68,96 pontos para a pontuaçáo total e média de 23,78 pontos para o escore motor, 22,5 para o atencional e 30,68 para o escore falta de planejamento.

Analisando os escores de impulsividade da escala de impulsividade de Barratt versão 11 para as fases iniciais, verificamos que estas obtiveram uma média de pontuação total de 68,76 pontos, com 23,34 pontos para o escore motor, 22,56 para o escore atencional e 30,8 pontos para o escore falta de planejamento.

Quando comparadas às fases finais dos mesmos cursos, foi evidenciado que a pontuação total destas na BIS 11 correspondeu a 69,74 pontos, com escores motor, atencional e falta de planejamento pontuando uma média de 25,08; 22,13 e 29,79 pontos respectivamente (tabela 2).

Tabela 2: Escala de impulsividade de Barrat BIS 11 - pontuação total e subdomínios classificada por curso e fases

\begin{tabular}{lcccc}
\hline BIS 11 & Direito & Engenharia & $\begin{array}{c}\text { Fases } \\
\text { Iniciais }\end{array}$ & $\begin{array}{c}\text { Fases } \\
\text { FUBnais }\end{array}$ \\
\hline Bis 11 - motor & 23,98 & 23,78 & 23,34 & 25,08 \\
Bis 11 - atencional & 22,33 & 22,5 & 22,56 & 22,13 \\
Bis 11 - falta de & 30,25 & 30,68 & 30,8 & 29,79 \\
planejamento & & & & \\
Bis 11 - total & 69,18 & 68,96 & 68,76 & 69,74 \\
\hline
\end{tabular}

Quanto à relação entre características sociodemográficas e comportamento impulsivo, verificou-se que $70,9 \%$ dos alunos pontuaram entre 52 e 71 , enquanto $29,1 \%$ pontuaram 72 ou mais pontos. A pontuação entre 52 e 71 pontos correspondeu a $67,6 \%$ dos alunos, enquanto $32,4 \%$ pontuaram 72 ou mais pontos.

$\mathrm{Na}$ relação entre os sexos, pode-se observar que homens e mulheres em sua maioria pontuaram entre 5271 pontos (44 e 45 respectivamente). Quanto ao curso, contatou-se que o de Engenharia Civil obteve maior frequência entre os que pontuaram 52-71 (50) e $\geq 72$ (74), sendo que a maioria dos alunos que obtiveram pontuação entre 52-71 estão nas fases inicias de ambos os cursos (61) e aqueles com pontuação $\geq 72$ nas fases finais (74).
Analisando as classes sociais, a pontuação entre 52 e 71 pontos para as classes $\mathrm{A}, \mathrm{B}, \mathrm{C}$ e $\mathrm{D}$ foi respectivamente de 28; 51; 9; e 1 aluno, totalizando 89 estudantes, enquanto a pontuação de 72 ou mais pontos correspondeu à 7; 28; 5; e zero para as classes A, B, C e D respectivamente, totalizando 40 alunos. No entanto, não houve diferença significativa entre as variáveis (tabela 3).

Tabela 3: Características sociodemográficas relacionadas ao comportamento impulsivo

\begin{tabular}{lccc}
\hline BIS & & & \\
\hline & $\begin{array}{c}52-71 \\
\text { pontos }\end{array}$ & $\geq \mathbf{7 2}$ pontos & $\begin{array}{c}\text { Valor } \\
\text { de p }\end{array}$ \\
\hline Gênero & & & 0,953 \\
\hline Masculino & 44 & 20 & \\
Feminino & 45 & 20 & \\
\hline Graduaçáo & & & 0,685 \\
\hline Direito & 39 & 55 & \\
Engenharia & 50 & 74 & \\
Fases & & & 0,651 \\
Iniciais & 61 & 29 & \\
Finais & 28 & 74 & \\
\hline Estrato econômico & & & 0,253 \\
\hline Estrato A & 28 & 7 & \\
Estrato B & 51 & 28 & \\
Estrato C & 10 & 5 & \\
\hline
\end{tabular}

\section{Discussão}

Com base nos resultados obtidos, observou-se que a maioria dos universitários pesquisados está dentro dos limites normais para a impulsividade. Entretanto, vale ressaltar que $31 \%$ pontuou $\geq 72$, o que classifica estes indivíduos como altamente impulsivos e que nenhum estudante desta amostra obteve escore menor que 52, sendo representativo de indivíduo que é muito controlado ${ }^{19}$. O resultado de Guera ${ }^{20}$, também em amostra de estudantes universitários, apresentou elevado índice de impulsividade, sobretudo motora e de planejamento ${ }^{20}$. Da mesma forma, outros estudos com populaçốes diferentes (alcoolistas, dependentes de drogas psicotrópicas, adolescentes), utilizando também a BIS 11, demonstrou na maior parte da amostra altos escores de impulsividade total ${ }^{21-24}$, o que difere do nosso estudo.

Foi verificado que homens e mulheres pesquisados possuem um escore de impulsividade total muito semelhante (69,02 e 69,09 respectivamente), assim como em todos os subdomínios. Entretanto, em outros estudos com estudantes universitários, observou-se diferença entre os sexos no escore de impulsividade, com níveis superiores para o sexo masculino ${ }^{25,26}$. Destaca-se que este fator pode estar relacionado a diferenças no sistema de neurotransmissão 
serotoninérgica menos presente nos homens ${ }^{27}$, o que é decisivo no momento do controle do impulso ${ }^{28}$.

$\mathrm{Na}$ relação impulsividade e curso de graduação, foi verificado que o curso de Direito ligado à área de Ciências Humanas e Sociais apresentou uma pontuação de impulsividade pouco menor relacionada ao curso de Engenharia Civil (Ciências Exatas e Tecnológicas), assim como em outro estudo com uma população de universitários de cursos distintos em que se observou menor índice de impulsividade no curso de Psicologia, também ligado à área de Ciências Humanas e Sociais. Porém, as diferenças não devem ser atribuídas apenas ao caráter do curso frequentado, visto que explicações alternativas, relacionadas ao sexo dos estudantes e às vivências na universidade, podem ser responsáveis por tais diferenças ${ }^{29}$.

Em alguns casos, a impulsividade na personalidade pode ser benéfica por levar a decisóes rápidas em momentos oportunos. No entanto, os componentes da impulsividade incluem atençáo, respostas suprimidas, pobre avaliação das consequências e/ou inabilidade de renunciar imediatamente pequenas recompensas em favor de recompensas maiores posteriores. $\mathrm{O}$ ato de decidir reflete um processo em que a atenção está focada e a decisão é tomada após a reflexão sobre possíveis desfechos e açóes. Este processo requer atençáo, embora com a repetição, este processo requeira menos e pode se tornar mais rápido, automatizado, mas náo impulsivo ${ }^{30}$.

Estudantes que ingressam na universidade estão expostos a diferentes questôes emocionais, que abalam seus sentimentos e comportamentos relacionados ao seu funcionamento diário ${ }^{31}$. De maneira geral, as condutas de impulsividade entre os estudantes deste estudo das fases iniciais e do final da graduação não apresentaram diferenças significativas. Porém, observou-se uma pontuação um pouco maior na média total da Bis 11 entre os estudantes do final do curso, representativo de maior impulsividade.

Quanto à classe socioeconômica dos alunos pesquisados, pode-se dizer que, em sua maioria, são elitizados, $88,3 \%$ dos estudantes pertencem às classes $\mathrm{A}$ e $\mathrm{B}$, segundo critérios da Associaçáo Brasileira de Empresas de Pesquisa (Abep) que envolve os que possuem renda familiar média acima de $\mathrm{R} \$ 2.327,00^{18}$, situação socioeconômica comum por se tratar de uma universidade particular. No entanto, quando feita a análise estatística comparando a pontuação da BIS 11 e a estratificação das classes sociais, obteve-se o valor de $p=0,347$, indicando que não há relação entre a impulsividade avaliada pela escala e a classe social dos alunos.

A impulsividade tem uma série de definiçóes que, generalizadamente, inclui ações que são pobremente refletidas, expressadas prematuramente, com riscos, ou inapropriadas para a situaçáo, podendo levar a consequências indesejáveis ${ }^{32}$. Diversos são os transtornos em que as diferentes manifestaçóes da impulsividade se apresentam de forma intensa, podendo levar a consequências indesejáveis, e gerar prejuízos para o indivíduo e aqueles que o cercam $^{2,32}$. No nosso estudo, as variáveis sociodemográficas não foram associadas com a impulsividade nos universitários, mas houve uma parcela considerável de alunos (31\%) classificados como altamente impulsivos.

\section{Conclusão}

Ao finalizar o estudo, observou-se que os universitários apresentaram na sua maioria um comportamento impulsivo dentro da normalidade, havendo um leve aumento da pontuaçáo entre os estudantes de fases finais quando comparados com estudantes de fases iniciais e daqueles que cursam Engenharia Civil. Quando relacionada à impulsividade entre os sexos, verificou-se que homens e mulheres se comportam de maneira semelhante. Contudo náo foi encontrada associação entre a impulsividade e as características sociodemográficas.

Sugerem-se novas pesquisas que busquem um diagnóstico mais precoce, permitindo a criação de estratégias de prevenção e enfrentamento da impulsividade, uma vez que o comportamento impulsivo em jovens está diretamente relacionado com a adição ao álcool, fumo, drogas psicotrópicas e acidentes automobilísticos.

Importante também buscarmos uma forma positiva de lidar com a impulsividade a fim de promover e valorizar uma vida de melhor qualidade para estes jovens, os quais serão os futuros profissionais em suas respectivas áreas.

\section{Referências}

1. Lajunen T, Parker D. Are aggressive people aggressive drivers? A study of the relationship between self-reported general aggressiveness, driver anger and aggressive driving. Accid Anal Prev. 2001; 33(2): 243-55.

2. Malloy-Diniz LF, Mattos P, Leite WB, Abreu N, Coutinho G, De Paula JJ, et al. Tradução e adaptação cultural da Barratt Impulsiveness Scale (BIS-11) para aplicação em adultos brasileiros. J Bras Psiquiatr. 2010; 59(2): 99-105.

3. Araújo MM, Malloy-Diniz LF, Rocha FL. Impulsividade e acidentes de trânsito: Revisão de Literatura. Rev Psiq Clin. 2009; 36(2): 60-8.

4. Villar I. Déficit de Atención con Hiperactividad: manual para padres y educadores. 1 ed. Madrid: CEPE; 1998.

5. Amen DG. Transforme seu cérebro, transforme sua vida Um programa avançado para vencer a ansiedade, a depressão, a obsessividade, a raiva e a impulsividade. Change your Brain, change your life, 1 ed. Fairfield: Mercuryo; 2000.

6. Scheffer M, Almeida RMM. Consumo de álcool e diferenças entre homens e mulheres: comportamento impulsivo, 
aspectos cognitivos e neuroquímicos. Rev Neuropsicologia Latinoamericana. 2010; 2(3): 1-11.

7. Tavares H. Jogo patológico - Impulsividade e jogos de azar. Jornal da Fundação Faculdade de Medicina. 2009 Jan-Fev; 3.

8. Prado-Lima PAS. Tratamento farmacológico da impulsividade e do comportamento agressivo. Rev Bras Psiquiatr. 2009; 31(2): 58-65.

9. Wellausen RS. Avaliação dos fatores associados ao uso de álcool e drogas na criminalidade: um estudo no sistema penitenciário [dissertação]. Porto Alegre (RS): Universidade Federal do Rio Grande do Sul; 2009.

10. Williams WA, Potenza MN. Neurobiologia dos transtornos do controle dos impulsos. Rev Bras Psiquiatr. 2008; 30(1): 24-30.

11. Potenza MN, Hollander E. Pathologic gambling and impulse control disorders. IN: Davis KL, Charney D, Coyle JT, NemeroffC, organizadores. Neuropsychopharmacology: the fifth generation of progress. Philadelphia: Lippincott Williams \& Wilkins; 2002: 1725-41.

12. Dornelles C. DSM-IV-TR Manual diagnóstico e estatístico de transtornos mentais (tradução), 4 ed. Texto revisado. São Paulo: Artmed; 2002.

13. Eric R, Martin RC, Rangan K, Kuhlman M. Driving anger, sensation seeking, impulsiveness and boredom proneness in prediction of unsafe driving. Accid Anal Prev. 2005; 37(2): 341-8.

14. Diemen LV, Szobot CM, Kessler F, Pechansky F. Adaptação e validade de constructo da escala de impulsividade de Barrat (BIS 11) para o português do Brasil para o uso em adolescentes. Rev Bras Psiquiatr. 2007; 29(2): 153-6.

15. Diemen LV. Associação entre impulsividade, idade do primeiro consumo de álcool e abuso de substâncias psicoativas em adolescentes de uma região do sul do Brasil [dissertação]. Porto Alegre (RS): Universidade Federal do Rio Grande do Sul; 2006.

16. Stoltenberg SF, Batien BD, Birgnheirr DG. Does gender moderate associations among impulsivity health-risk behaviors? Addictive Behaviors. 2008; 33(2): 252-65.

17. ABEP. Associação Nacional de Empresas de Pesquisa. Critério de classificação econômica Brasil. São Paulo: Associaçáo Nacional de Empresas de Pesquisa; 2010.

18. Stanford MS, Mathias CW, Dougherty DM, Lake SL, Anderson NE, Patton JH. Fifty years of the Barratt Impulsiveness Scale: an update and review, Personality and Individual Differences: The Official Journal of the International Society for the Study of Individual Differences. 2009; 47(5): 385-95.

19. Stanford MS, Mathias CW, Dougherty DM, Lake SL, Anderson NE, Patton JH. Fifty years of the Barratt
Impulsiveness Scale: An update and review. Pers Individ Dif. 2009; 47(5): 385-95.

20. Guera LSM. Preditores da Compulsividade Sexual: afeto, impulsividade e alexitimia [dissertação]. Aveiro: Universidade de Aveiro; 2012.

21. Bjork JM, Hommer DW, Grant SJ, Danube C. Impulsivity in Abstinent Alcohol-dependent patients: relation to control subjects and type 1-/type 2-like traits. Alcohol. 2004; 34(2-3): 133-50.

22. Von-Diemen L, Bassani DG, Fuchs SC, Szobot CM, Pechansky F. Impulsividade, a idade do primeiro uso do álcool e de substâncias, distúrbios entre adolescentes do sexo masculino: uma população com base no estudo caso-controle. Addiction. 2008; 103(7): 1198-205.

23. Costa LLS, Navas ALGP, Oliveira CCC, Ratto LRC, Carvalho KHP, Silva HR, et al. Avaliação da memória operacional fonológica e impulsividade de usuários de drogas atendidos em um centro de atenção integrada à saúde mental. Rev CEFAC 2012; 14(3): 438-47.

24. Nandagopal JJ, Fleck DE, Adler CM, Mills NP, Strakowski SM, DelBello MP. Impulsivity in Adolescents with Bipolar Disorder and/or Attention-Deficit/Hyperactivity Disorder and Healthy Controls as Measured by the Barratt Impulsiveness Scale. J Child Adolesc Psychiatr. 2011; 21(5): 465-8.

25. Adan A, Natale V, Caci H, Prat G. Relationship between circadian typology and functional and dysfunctional impulsivity. Chronobiol Int. 2010; 27: 606-19.

26. Adan A. Functional and dysfunctional impulsivity in young binge drinkers. Adicciones. 2012; 24(1): 17-22.

27. Verdejo A, Lawrence AJ, Clark L. Impulsivity as a vulnerability marker for substance-use disorders: review of findings from high-risk research, problem gamblers and genetic association studies. Neurosci Biobehav Rev. 2008; 32(4): 777-810.

28. Strüber D, Lück M, Roth J. Sex, aggression and impulse control: an integrative account. Neurocase. 2008; 14(1): 93-121.

29. Martins RMM, Santos AAA, Dib Bariani. Estilos cognitivos e compreensão leitora em universitários. Paideia. 2005; 15(30): 57-68.

30. Congdon E, Canli T. A neurogenetic approach to impulsivity. J Pers. 2008; 76:1447-84.

31. Catunda MAP, Ruiz VM. Qualidade de vida de universitários. Pensamento Plural: Rev Científica UNIFAE. 2008; 2(1): 22-31.

32. Wit H. Impulsivity as a determinant and consequence of drug use: a review of underlying processes. Addict Biol. 2009; 14:22-31. 